NEUROLOGICAL PICTURE

\title{
Multiple hydatid cysts of the brain after surgery
}
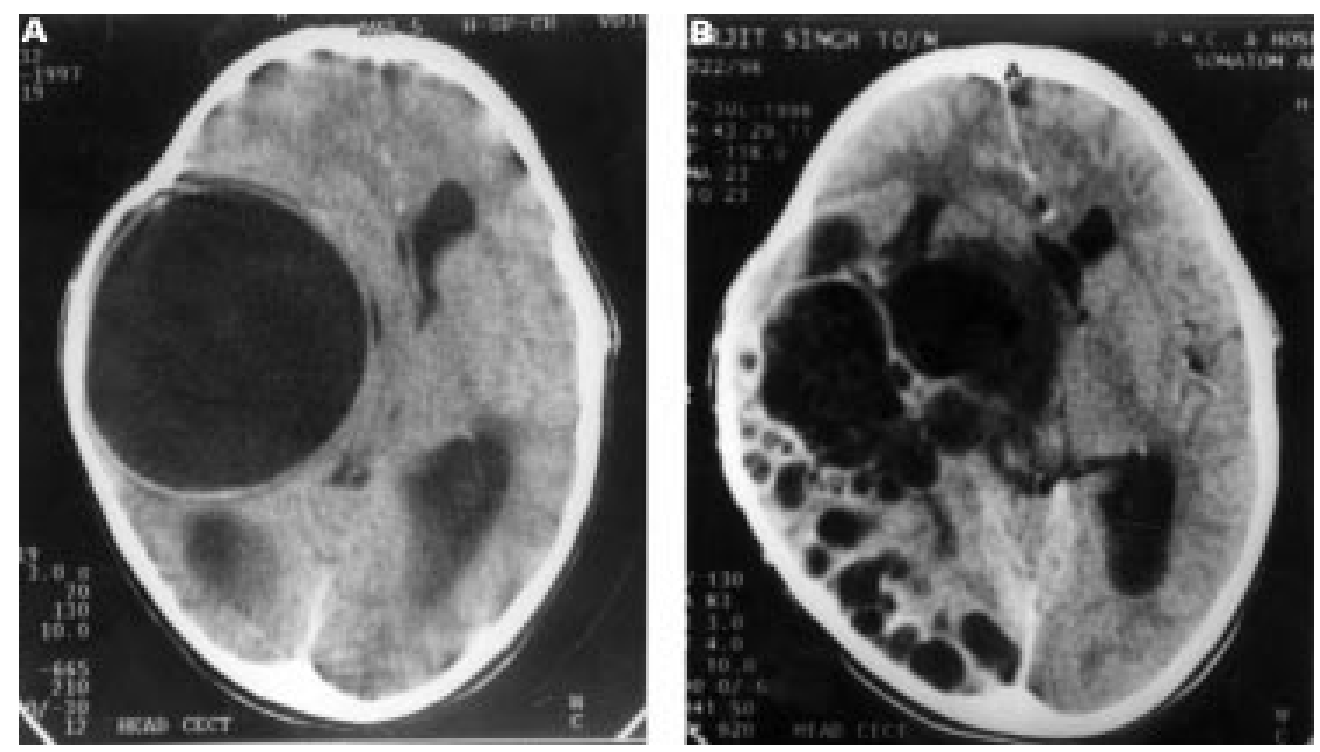

(A) Preoperative CT shows a large unilocular cyst in the right cerebral hemisphere. (B) CT 8 months after the operation shows new and multiple attached cysts, presumably because of rupture during the operation.

A 10 year old boy presented with complaints of fever, headache, and vomiting for 5 days. He had a history of craniotomy for removal of a benign cyst on the right side, 8 months previously (figure A). Review of previous CT showed a smooth walled cystic space occupying besion roughly $8 \mathrm{~cm}$ in diameter in the right temporoparietal region. There was effacement of the ipsilateral ventricle with midline shift of $18 \mathrm{~mm}$ to left. No evidence of calcification or surrounding oedema was seen. The skull vault was thinned out, showing an obvious bulge. On contrast enhanced CT the lesion did not take up any contrast, but was better defined. Surgical notes showed a large white, thick walled, cystic mass roughly $7.5 \mathrm{~cm}$ in diameter which was excised. Histopathology showed it to be an hydatid cyst.

Brain CT at the second admission showed new and multiple attached cystic lesions in the right cerebral hemisphere (figure B). The larger cyst showed internal septations suggestive of daughter cysts. A moderate amount of surrounding oedema was seen. Right lateral and third ventricles were compressed and pushed to the left. The patient was treated conservatively.

$$
\begin{array}{r}
\text { PARAMBIR SANDHU } \\
\text { KAVITA SAGGAR } \\
\text { KUSHALJT SINGH SODHI } \\
\text { Department of Radiodiagnosis, Dayanand Medical College and Hospital Ludhiana, India }
\end{array}
$$

